\title{
Ultrafast Transmission Electron Microscopy with High-Coherence Electron Pulses
}

Tyler Harvey ${ }^{1}$, Armin Feist ${ }^{1}$, Marcel Möller ${ }^{1}$, Nara Rubiano da Silva ${ }^{1}$, Nora Bach ${ }^{1}$, Till Domröse ${ }^{1}$, Thomas Danz ${ }^{1}$, John Gaida ${ }^{1}$, Thomas Rittman ${ }^{1}$, Katharina Priebe ${ }^{1}$, Sascha Schäfer ${ }^{1,2}$, Claus Ropers ${ }^{1}$

1. 4th Physical Institute, University of Göttingen, Göttingen, Germany

2. Institute of Physics, University of Oldenburg, Oldenburg, Germany

Transmission electron microscopes typically offer excellent spatial resolution, but limited temporal resolution. It is possible to infer much about the behavior of materials based on a combination of atomicscale structural and chemical information and bulk or lower-resolution observations of the functional properties of materials. We can learn more by directly imaging nanoscale dynamics. Ultrafast transmission electron microscopy (UTEM) offers this capability using stroboscopic imaging, where an a femtosecond pulse of electrons probes dynamics pumped by an optical pulse or electrical signal. The first ultrafast transmission electron microscopes [1-2] employed a flat photocathode and electron pulses with a subsequently large effective source size. These instruments brought femtosecond time resolution to the transmission electron microscope, but sacrificed spatial resolution to do so. We report on the linear photoemission from needle-shaped photocathodes for which the emission is confined to nanometer-sized areas, resulting in highly coherent electron pulses [3-6]. We illustrate that the combined nanometer spatial and femtosecond temporal resolution of this instrument can be used to image lattice dynamics, probe resonances in magnetic nanostructures, and observe ultrafast demagnetization in real space.

The Göttingen UTEM employs single-photon photoemission to generate electron pulses from a laserdriven Schottky field-emitter. The emitter is based on a single-crystalline (100)-oriented tungsten needle, which is covered with a zirconium oxide overlayer. Schottky emitters are usually operated at an elevated temperature of about $1800 \mathrm{~K}$ and an applied electric field in the range of $0.5-1 \mathrm{~V} / \mathrm{nm}$ [7]. For a (100)-oriented apex facet, the $\mathrm{ZrO}$ overlayer results in a selective workfunction reduction, supporting tunneling-assisted thermal electron emission at moderate temperatures. For their use as photocathodes, the facet-specific reduction of the work function can be utilized to trigger localized linear photoemission from the tip apex. Specifically, we operate the photocathode at temperature low enough to extinguish continuous thermal emission. We then illuminate the apex with femtosecond laser pulses at a central wavelength of $400 \mathrm{~nm}$ (see Fig. 1b). A linear scaling of the photoelectron current with the incident optical fluence is observed, allowing for a flexible tuning of the temporal electron pulse structure. The photoelectron pulses are accelerated to $120 \mathrm{keV}$ in a JEOL 2100F. In the space-charge-free regime, the electron pulses show a narrow spectral width of $0.6 \mathrm{eV}$ and a pulse duration of $200 \mathrm{fs}$ (full-width-at-halfmaximum, characterized by electron-light interferometry [8]) (see Fig. 1e,f). We have measured a minimum electron probe size of below $1 \mathrm{~nm}$ (Fig. 1c) and a beam emittance down to $1.8 \mathrm{~nm}-\mathrm{mrad}$, and a degree of transverse coherence exceeding $10 \%$, calculated from the interference fringes generated by a Möllenstedt biprism provided by the Lichte group in Dresden (Fig. 1d) [8].

These exceptional beam properties allow for both coherent manipulation of the electron quantum state to produce electron pulse trains into attosecond bursts [9], as well as observation of structural, electronic and magnetic dynamics on the nm-fs scale. In first experiments (Fig. 2), we demonstrated the broad applicability of ultrashort electron pulses in high-resolution imaging and diffraction, Lorentz microscopy, electron energy loss spectroscopy and scanning diffraction [10]. These capabilities allow us 


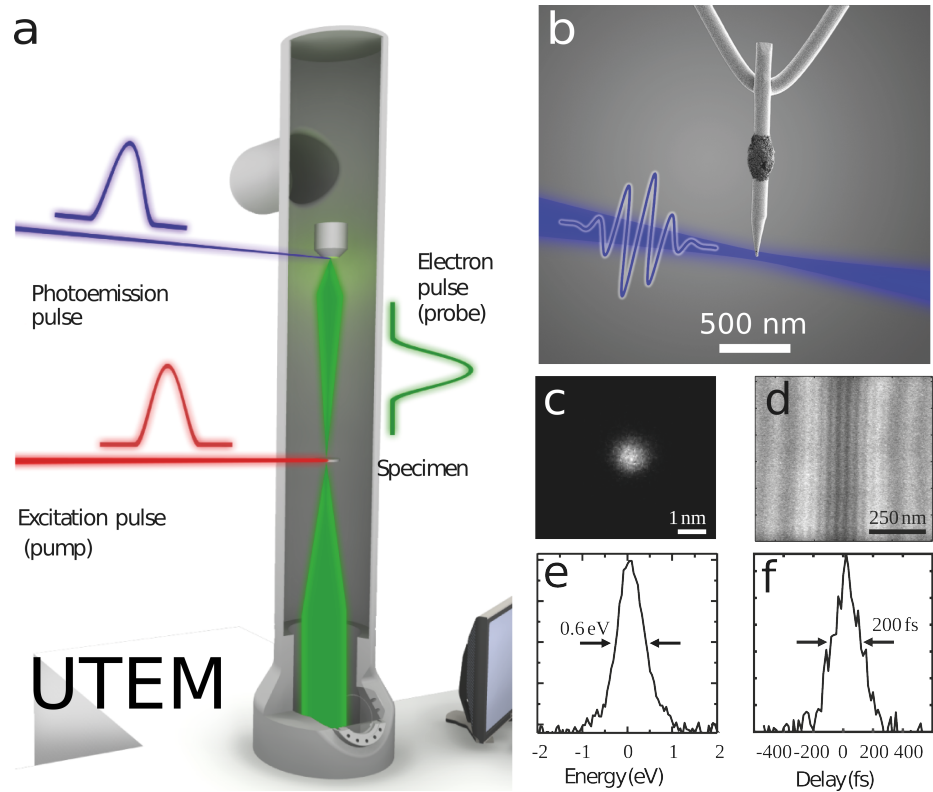

Figure 1. (a) Schematic of Göttingen UTEM, with stroboscopic laser pump and electron probe principle. (b) Photoelectron pulses are generated by illumination of a heated tungsten tip emitter with a $\mathrm{ZrO} 2$ reservoir. (c) Minimum electron spot size. (d) Photoelectron hologram measured with a biprism (adapted from [8]). $(\mathrm{e}, \mathrm{f})$ Measured optimal spectral and temporal width.

to, for example, map strain dynamics and probe nanoscale magnetic dynamics.

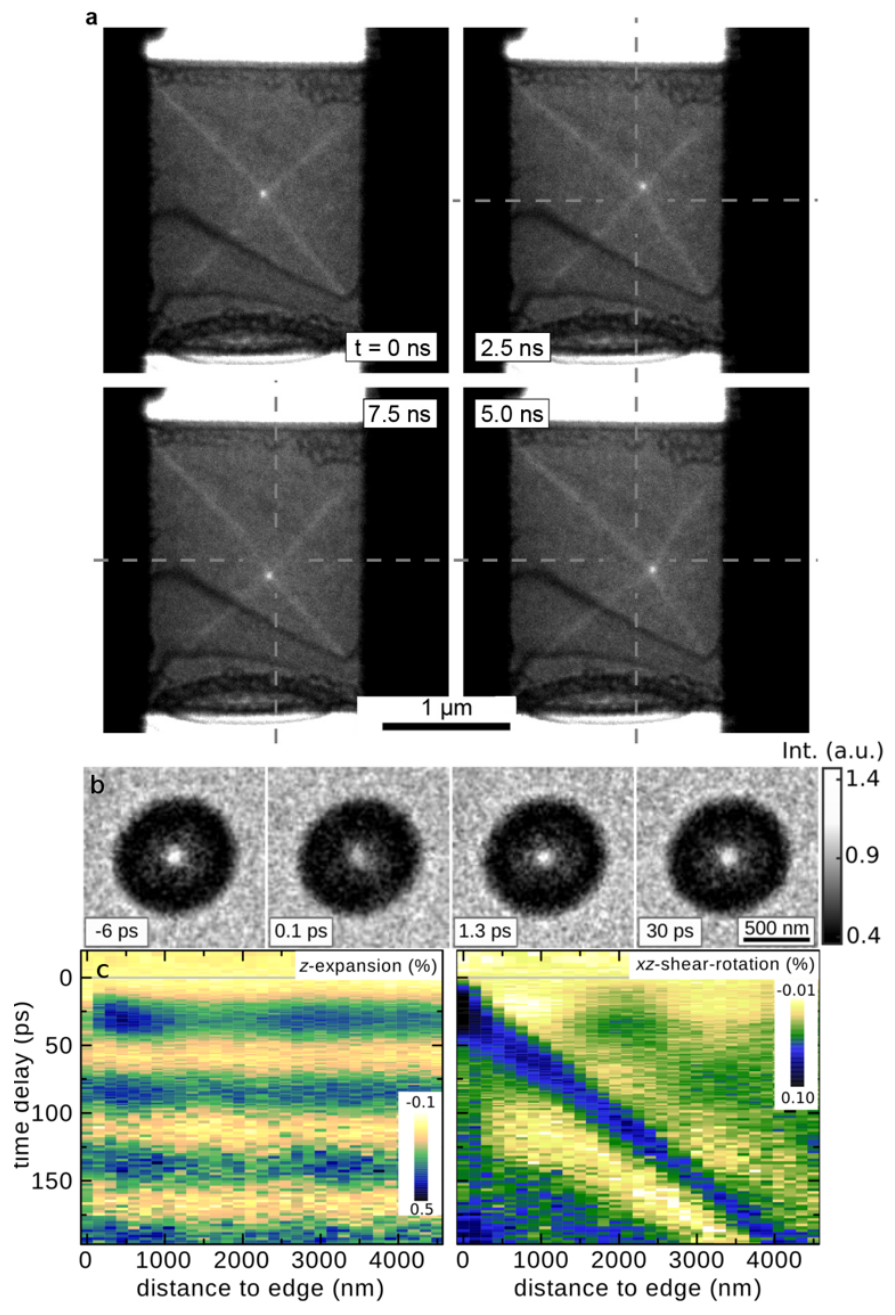

References:

[1] H. Dömer, and O. Bostanjoglo, Rev. Sci. Instrum. 74, 4369 (2003).

[2] A. H. Zewail, Science 328, 187 (2010).

[3] P. Hommelhoff, C. Kealhofer, and M. Kasevich, Phys. Rev. Lett. 97, 24740 (2006).

[4] C. Ropers, D. R. Solli, C. P. Schulz, C. Lienau, and T. Elsaesser, Phys. Rev. Lett. 98, 043907 (2007).

[5] A. Paarmann, M. Gulde, M. Müller, S. Schäfer, S. Schweda, M. Maiti, C. Xu, T. Hohage, F. Schenk, C. Ropers, and R. Ernstorfer, J. Appl. Phys. 112, 113109 (2012).

[6] B. Cook, M. Bronsgeest, K. Hagen, and P. Kruit, Ultramicroscopy 109, 403 (2009).

[7] M. Fransen, M.H.F. Overwijk, and P. Kruit, Appl. Surf. Sci. 146, 357 (1999).

[8] A. Feist et al., Ultramicroscopy 176, 63 (2017).

[9] K. E. Priebe et al., Nature Photonics 11, 793 (2017).

[10] A. Feist, N. Rubiano da Silva, W. Liang, C. Ropers, and S. Schäfer, Struct. Dyn. 5, 14302 (2018).

[11] N. Rubiano da Silva, M. Möller, A. Feist, H. Ulrichs, C. Ropers and S. Schäfer, arXiv:1710.03307. 